\title{
Die Ärzteschaft ist offen für neue Finanzierungsmodelle
}

\author{
Beatrix Meyer ${ }^{a}$, Cloe Jans ${ }^{b}$, Lukas Golder ${ }^{c}$
}

${ }^{a}$ Leiterin Abteilung Stationäre Versorgung und Tarife, FMH; ${ }^{b}$ Projektleiterin gfs.bern; ${ }^{\mathrm{c}}$ Co-Leiter gfs.bern

Die Ärztinnen und Ärzte befürworten eine einheitliche Finanzierung im ambulanten und stationären Sektor. Dadurch könnten Fehlanreize reduziert werden. Zunehmend belastet werden die Ärzte durch den stetig steigenden Dokumentationsaufwand: Die Spitalärzte der Akutsomatik verbringen durchschnittlich pro Tag 26 Minuten mehr mit Dokumentieren als im Jahr 2011.

Seit sechs Jahren rechnen die Spitäler in der stationären Akutsomatik mit SwissDRG ab. Die Leistungen der stationären Erwachsenenpsychiatrie werden seit Anfang 2018 mit TARPSY vergütet. Für die stationäre Rehabilitation ist die Einführung des Tarifsystems ST Reha im Jahr 2020 geplant. Zudem stehen umfangreiche Änderungen des Finanzierungssystems des Schweizer Gesundheitswesens zur Diskussion. Um allfällige Fehlentwicklungen frühzeitig zu erkennen, führt das Forschungsinstitut gfs.bern im Auftrag der FMH seit 2011 jährliche repräsentative Befragungen durch. An der jüngsten Umfrage im Sommer 2017 haben 1471 Ärztinnen und Ärzte teilgenommen. ${ }^{1}$

\section{Zustimmung zu einheitlicher Finanzie- rung stationär - ambulant}

Abgesehen vom Selbstbehalt werden heute die ambulanten Leistungen durch die Versicherer alleine vergütet. Im stationären Sektor bezahlen die Kantone hingegen mindestens $55 \%$ bei Leistungen der Listenspitäler. Deshalb ist es für die Versicherer oft kostengünstiger, wenn der Patient stationär statt ambulant behandelt wird. Es bestehen also Fehlanreize, die das Schweizer Gesundheitswesen insgesamt verteuern. Die akutsomatisch tätigen Spitalärzte stellten in ihrem Arbeitsbereich durchschnittlich 4,4 Mal pro Monat fest, dass der Entscheid spitalambulant vs. stationär nicht aus medizinischen Gründen erfolgte. Eine relative Mehrheit der befragten Ärztinnen und Ärzte ist denn auch mit der heutigen Finanzierung nicht zufrieden. Das Bedürfnis nach neuen Lösungen ist also durchaus spürbar. Eine einheitliche Finanzierung sowohl im stationären als auch im ambulanten Bereich geniesst dabei mit Abstand die grösste Zustimmung. Die Kantone würden sich dabei sowohl im ambulanten als auch im stationären Sektor gleichermassen beteiligen, beispielsweise mit einem Finanzierungsanteil von $25 \%$. $32 \%$ der praxisambulant tätigen Ärzte sowie $37-44 \%$ der in der Rehabilitation, Akutsomatik und Psychiatrie engagierten Spitalärzte befürworten diese Lösung.

Ärzte wünschen im ambulanten Sektor die Mitfinanzierung durch die Kantone.

Eine monistische Finanzierung allein durch die Versicherer findet hingegen ebenso wenig Akzeptanz wie das heutige Modell. Jeder sechste Befragte ist von keiner dieser Lösungen überzeugt oder hat andere Ideen. Rund ein Viertel der Befragten ist unentschlossen oder äusserte sich nicht dazu (vgl. Abb. 1).

\section{Weiterhin Vorbehalte gegenüber Swiss- DRG und TARPSY}

Eine relative Mehrheit der Ärzteschaft wünscht sich zwar einen Wechsel der Finanzierungsmodelle, das heisst eine Änderung, wer die Spitalrechnung bezahlt. Vorbehalte haben die Ärztinnen und Ärzte hingegen weiterhin gegenüber den Tarifsystemen. Knapp die Hälfte der jeweils betroffenen Ärzte steht den Tarifsystemen SwissDRG und TARPSY klar oder eher ablehnend gegenüber. Weniger Vorbehalte sind in der Rehabilitation zu verzeichnen: Rund ein Viertel der in der stationären Rehabilitation tätigen Ärzte steht der Ein-

\section{Neue Finanzierungsmodelle}

Weitere Informationen zu neuen Finanzierungsmodellen: www. fmh.ch $\rightarrow$ StationäreTarife $\rightarrow$ Publikationen $\rightarrow$ FMH-Tagung: Neue Wege der Finanzierung im Gesundheitswesen. 
"Welches Finanzierungssystem wünschen Sie sich am meisten für die Zukunft?" in \% ambulant tätige Ärzte/akutsomatische Ärzte/Psychiatrie/Rehabilitation

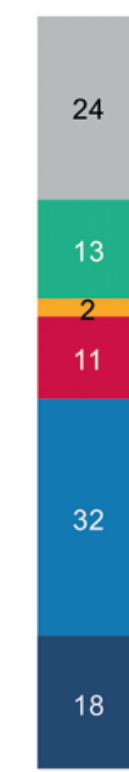

ambulant tätige Ärzte

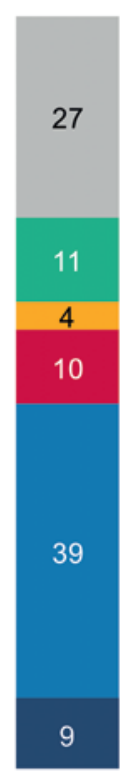

akutsomatische Ärzte

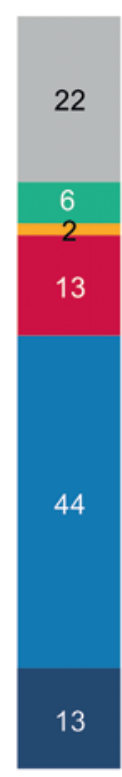

Psychiatrie

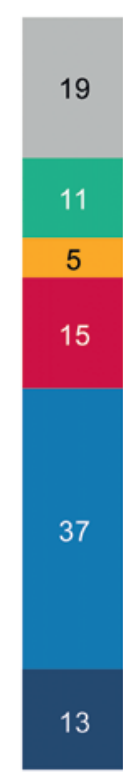

Rehabilitation weiss nicht/keine Antwort

keine dieser Ideen überzeugt

anderes

- Finanzierung in ambulanten und stationären Sektor allein durch Versicherer

- Kantone beteiligen sich im stationären und im ambulanten Sektor

- gleich wie heute

C gfs.bern, Begleitstudie im Auftrag der FMH, Juni - August 2017 (N ambulant tätige Ärzte $=295$ /

$\mathrm{N}$ akutsomatische Ärzte $=974 / \mathrm{N}$ Psychiatrie $=127 / \mathrm{N}$ Rehabilitation $=75)$, sig.

Abbildung 1: Gewünschtes Finanzierungssystem.

führung von ST Reha klar oder eher ablehnend gegenüber. Viele haben sich aber noch keine Meinung dazu gebildet. Dies könnte damit zusammenhängen, dass noch offen ist, wie genau die Einführungsversion ST Reha ausgestaltet sein wird.

\section{Steigender Dokumentationsaufwand - weniger Zeit für Patienten}

Zunehmend belastet werden die Ärztinnen und Ärzte unter anderem durch den stetig steigenden Dokumentationsaufwand. Die Spitalärztinnen und -ärzte in der Akutsomatik verbringen durchschnittlich 19\% ihrer Arbeitszeit (vgl. Abb. 2) bzw. 112 Minuten pro Tag mit Dokumentationsarbeiten; das sind 26 Minuten pro Tag mehr als im Jahr 2011. In der Psychiatrie beträgt der Anteil der Dokumentationsarbeit 15\% und in der Rehabilitation 18\%. Für die Patientinnen und Patienten

\section{Dokumentationsmuster}

Zur Vereinfachung der Dokumentation hat die FMH Dokumentationsmuster für Komplexcodes erstellt, neu auch für die Psychiatrie: www.fmh.ch $\rightarrow$ Stationäre Tarife $\rightarrow$ SwissDRG $\rightarrow$ Dokumentationsmuster. bleibt damit immer weniger Zeit: Die patientennahen Tätigkeiten machen in der Akutsomatik und in der Psychiatrie im Schnitt nur noch rund einen Drittel der Arbeitszeit aus, in der Rehabilitation nur rund einen Fünftel. Betroffen von der Dokumentationsarbeit sind insbesondere die Assistenzärzte: Sie verbringen in der Akutsomatik 28\% ihrer Arbeitszeit mit Dokumentationsarbeiten und nur 30\% mit patientennahen Tätigkeiten.

\section{Arbeitszufriedenheit sinkt trotz starker Berufsidentifikation}

Die grosse Mehrheit der Ärztinnen und Ärzte identifiziert sich stark mit ihrem Beruf und schätzt die Teamarbeit insbesondere auch mit der Pflege. Rund 80\% der akutsomatisch und psychiatrisch engagierten Spitalärzte sind zufrieden mit ihrer Arbeitstätigkeit. In der Rehabilitation sind es 75\%. Am zufriedensten mit ihrer Tätigkeit sind mit 87\% die Ärzte in der ambulanten Praxis. Allerdings sinken die Zufriedenheitswerte in allen genannten Bereichen seit 2011. Besonders zu beobachten ist dies in der Psychiatrie und in der Rehabilitation; dort sank die Zufriedenheit seither um rund 10 Prozentpunkte. Beeinträchtigt wird die Zufrieden- 
"Wenn Sie an den letzten normalen Arbeitstag denken, wie viel Zeit haben Sie dabei auf die folgenden Tätigkeiten angewendet? Bitte geben Sie Ihre Angaben in Minuten an."

Ärztliche Dokumentationsarbeit/Patientendossier/Berichte verfassen

in \% Mittelwerte einzelner Tätigkeiten an gesamtem Arbeitstag, akutsomatische Ärzte
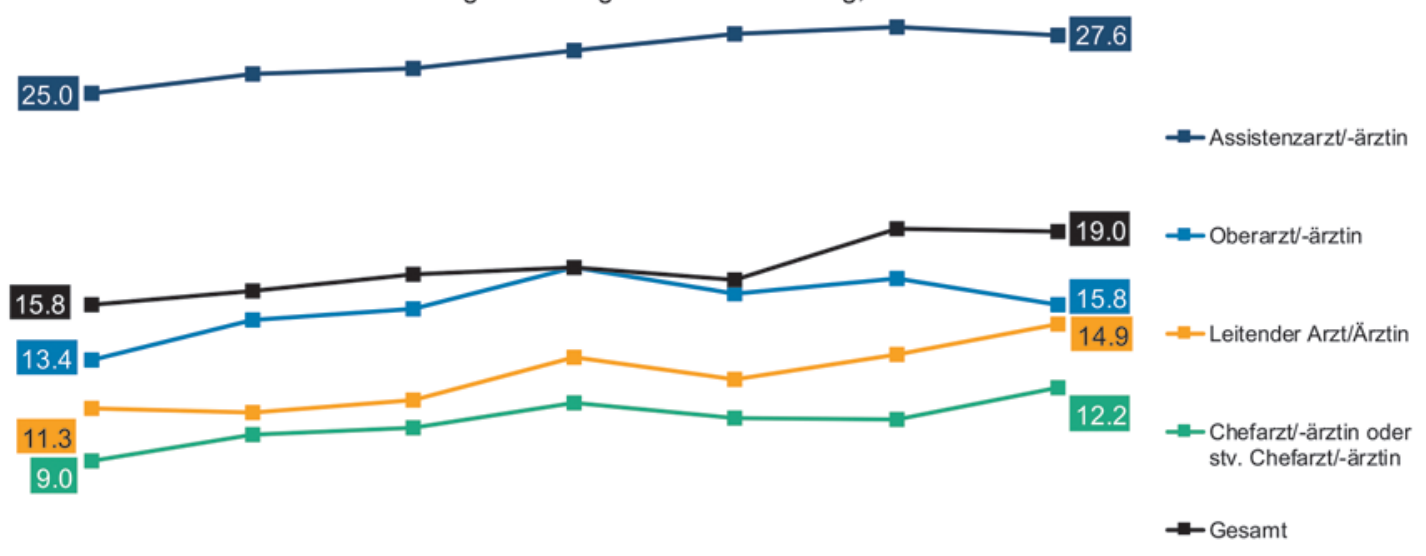

$\begin{array}{lllllll}2011 & 2012 & 2013 & 2014 & 2015 & 2016 & 2017\end{array}$

(c) gfs.bern, Begleitstudie im Auftrag der FMH, Juni - August 2017

( $N$ akutsomatische Ärzte jeweils ca. 940)

Abbildung 2: Zeitaufwand für ärztliche Dokumentationsarbeit.

heit oft durch den hohen Zeit- und Leistungsdruck, aber teilweise auch durch eine schlechte Arbeitsorganisation im Spital. So bemängeln 36\% der Spitalärzte die Arbeitsorganisation in ihrem Spital. Die Überstunden betragen bei Vollzeitstellen im Durchschnitt 7,4 Stunden pro Woche. In der Akutsomatik und Rehabilitation denken deutlich mehr Spitalärzte an eine Stelle ausserhalb des schweizerischen Gesundheitswesens nach als im Jahr 2013. Heute sind es in der Akutsomatik bereits 16\% und in der Rehabilitation 17\%. In der stationären Psychiatrie sind es 9\% und in der Praxisambulanz konstante 6\%.

\section{Boni in der Psychiatrie und Rehabili- tation auf dem Vormarsch}

Rund ein Drittel der Spitalärztinnen und -ärzte werden mit variablen Lohnkomponenten vergütet. Im Vordergrund stehen dabei zielbezogene Boni und privatärztliche Honorare. 9\% der in der Akutsomatik tätigen Spitalärzte beziehen einen zielbezogenen Bonus. Dieser Anteil ist im Vergleich zu den beiden Vorjahren leicht zurückgegangen. In der Psychiatrie und Rehabilitation hingegen sind die Boni auf dem Vormarsch. In der Psychiatrie beziehen 14\% und in der Rehabilitation 15\% einen zielbezogenen Bonus (vgl. Abb. 3). Sofern ein Spitalarzt zielbezogene Boni bezieht, betragen diese in der Akutsomatik durchschnittlich 17\% seines Gesamtlohnes. Im Jahr 2013 waren es noch 20\%. Spitalarztverträge mit Bonusvereinbarungen können insbesondere

\section{Die Spitalärzte der Akutsomatik erhalten} weniger Boni.

dann problematisch sein, wenn die Ziele an die Anzahl Behandlungen geknüpft sind. ${ }^{2}$ Die befragten Spitalärzte der Akutsomatik beobachteten in ihrem Arbeitsumfeld im Monat durchschnittlich 1,5 Operationen sowie 2,3 nicht-operative Behandlungen, die nicht medizinisch indiziert sind. Allerdings stellten die Spitalärzte auch fest, dass im Monat durchschnittlich zwei Behandlungen aus Kostengründen nicht durchgeführt wurden.

\section{Gute Qualität mit einigen Fehl- entwicklungen}

Eine deutliche Mehrheit der Spitalärzte bewertet die Patientenversorgung in ihrem unmittelbaren Arbeitsbereich als sehr gut oder gut. Im Vergleich zu 2012 beobachten in der Akutsomatik weniger Spitalärzte eine Verschiebung von multimorbiden oder chronisch kranken Patienten in andere Einrichtungen. Der Spielraum für die Behandlung wird nach wie vor mehrheit- 
Frage 1 "Existieren auf Ihrer Abteilung/in Ihrer Klinik Entschädigungssysteme mit variablen, leistungsabhängigen Lohnkomponenten?"

Frage 2 "Enthält Ihr persönliches Entschädigungssystem eine variable, leistungsabhängige Lohnkomponente?"

Frage 3 (falls variable, leistungsabhängige Lohnkomponente) "Handelt es sich bei der variablen, leistungsabhängigen Lohnkomponente um:"

in \% akutsomatische Ärzte/Psychiatrie/Rehabilitation
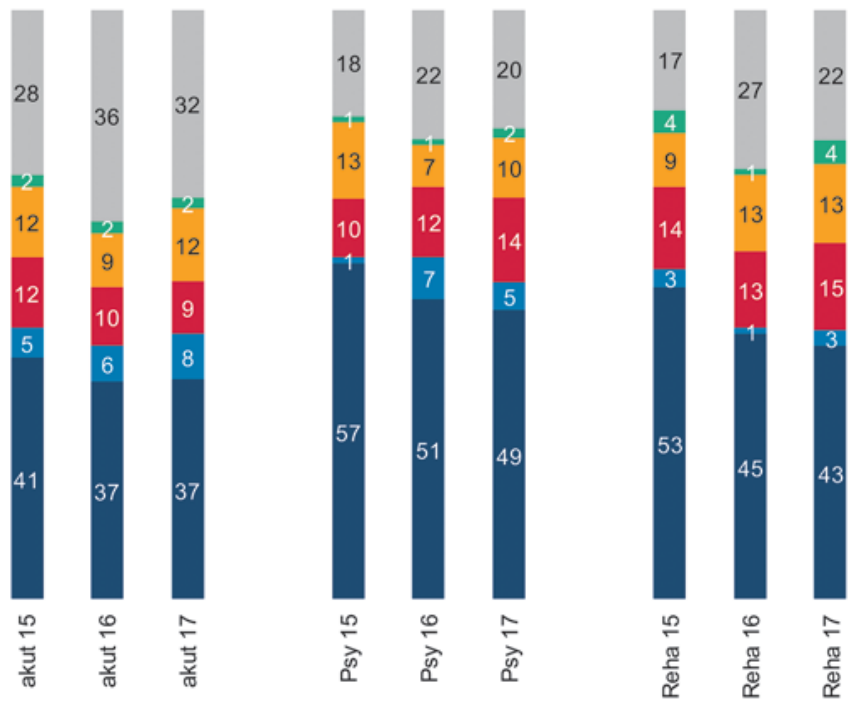

= keine Angabe bei Fragen
1,2 und 3
= ausschliesslich andere leistungsabhängige
Lohnkomponenten
= Einkünfte aus privatärztlicher
(Honorar-)Tätigkeit (teilweise kombiniert
mit anderen leistungsab.
Lohnkomponenten)
- Bonuszahlungen (teilweise kombiniert mit
anderen leistungsabhängigen
Lohnkomponenten)
= keine persönliche leistungsabhängige
Lohnkomponente

(c) gfs.bern, Begleitstudie im Auftrag der FMH, Juni - August 2017

(N akutsomatische Ärzte jeweils ca. 940 / N Psychiatrie jeweils ca. 130 / N Rehabilitation jeweils ca. 70)

Abbildung 3: Leistungsabhängige Lohnkomponenten.

lich als eher oder sehr gross bezeichnet. Wie in den Vorjahren sind jedoch $15 \%$ der akutsomatisch tätigen Ärzte der Ansicht, dass die Krankenkassen einen starken oder sehr starken Einfluss auf die Behandlung haben. In der Rehabilitation sind es $40 \%$ und in der Psychiatrie 26\%. Die in der Rehabilitation tätigen Spitalärzte stellten in ihrem Arbeitsbereich im Jahr 2017 durchschnittlich siebenmal pro Monat fest, dass Krankenkassen die Verlegung in die Rehabilitationsklinik verzögerten. $24 \%$ der praxisambulant tätigen Ärzte finden den Entlassungszeitpunkt aus den Spitälern der Akutsomatik häufig zu früh.

Korrespondenz: FMH

Baslerstrasse 47 CH-4600 Olten Tel. 0313591111 Fax 0313591112 tarife.spital[at]fmh.ch

\section{Weiter beobachten - unterwünschten Auswirkungen entgegenwirken}

Unabhängig davon, welche der zurzeit diskutierten Änderungen im Gesundheitswesen umgesetzt werden
- eine frühzeitige und langfristig angelegte Untersuchung möglicher Auswirkungen ist dabei wichtig. Denn unerwünschte Begleiterscheinungen wie beispielsweise der steigende administrative Aufwand verursachen hohe Kosten. Eine Optimierung der Prozesse und eine gute Zusammenarbeit mit den Krankenkassen sind deshalb unerlässlich. Dies ist nicht nur für die Zufriedenheit der Ärztinnen und Ärzte von höchster Bedeutung, sondern kommt letztlich auch den Patienten zugute.

\section{Fortbildungsseminare}

$\mathrm{FMH}$ und $\mathrm{H}+$-Bildung bieten ihre Seminare aufgrund grosser Nachfrage erneut an:

Seminarreihe Führung und Management für Ärztinnen und Ärzte Medizin und Ökonomie: Intensivseminar für Ärztinnen und Ärzte Weiterführende Informationen finden Sie auf www.fmh.ch $\rightarrow$ StationäreTarife. 